\section{Porphyromonas gingivalis lipopolysaccharide stimulation in human periodontal ligament stem cells: Role of epigenetic modifications to the inflammation}

\author{
Francesca Diomede, ${ }^{1}$ \\ Soundara Rajan Thangavelu, ${ }^{2}$ \\ Ilaria Merciaro, ${ }^{1}$ Monica D'Orazio, ${ }^{3}$ \\ Placido Bramanti, ${ }^{2}$ Emanuela Mazzon, ${ }^{2}$ \\ Oriana Trubiani ${ }^{1}$ \\ ${ }^{1}$ Stem Cells and Regenerative Medicine \\ Laboratory, Department of Medical, \\ Oral and Biotechnological Sciences, \\ University "G. d'Annunzio" Chieti- \\ Pescara, Chieti, Italy \\ ${ }^{2}$ IRCCS Centro Neurolesi "Bonino \\ Pulejo", Messina, Italy \\ ${ }^{3}$ Department of Rheumatology and \\ Immunology, Medical University of \\ Graz, Austria
}

\begin{abstract}
Periodontitis is a chronic oral inflammatory disease produced by bacteria. Gingival retraction and bone and connective tissues resorption are the hallmarks of this disease. Chronic periodontitis may contribute to the risk of onset or progression of neuroinflammatory pathological conditions, such as Alzheimer's disease. The main goal of the present study was to investigate if the role of epigenetic modulations is involved in periodontitis using human periodontal ligament stem cells (hPDLSCs) as an in vitro model system. hPDLSCs were treated with lipopolysaccharide of Porphyromonas gingivalis and the expression of proteins associated with DNA methylation and histone acetylation, such as DNMT1 and p300, respectively, and inflammatory transcription factor NF-kB, were examined. Immunofluorescence, Western blot and next generation sequencing results demonstrated that $P$. gingivalis lipopolysaccharide significantly reduced DNA methylase DNMT1, while it markedly upregulated the level of histone acetyltransferase p300 and NF-kB in hPDLSCs. Our results showed that $P$. gingivalis lipopolysaccharide markedly regulate the genes involved in epigenetic mechanism, which may result in inflammation induction. We propose that $P$. gingivalis lipopolysaccharide-treated hPDLSCs could be a potential in vitro model system to study epigenetics modulations associated with
\end{abstract}

periodontitis, which might be helpful to identify novel biomarkers linked to this oral inflammatory disease.

\section{Introduction}

Periodontal disease is a chronic inflammatory disease caused by sub gingival bacterial biofilm. ${ }^{1}$ This biofilm formation causes gingival retraction and bone and connective tissue resorption. ${ }^{2}$ Moreover, it has been demonstrated that in chronic condition inflammatory modulators associated with periodontitis may cause systemic inflammation, participate in the onset or in the progression of neuroinflammatory conditions, including Alzheimer's disease (AD) and produce cognitive decline. ${ }^{3-5}$ Although the real mechanism underlying the destruction of tooth supporting structures is not completely understood, bacterial interaction with the cells present in the oral cavity could be considered as a key pathogenetic step followed by biofilm synthesis. Moreover, mesenchymal stem cells (MSCs) present in the oral cavity are affected by bacteria and bacterial derivatives. ${ }^{6}$ Different types of dental MSCs have been reported: dental pulp stem cells, ${ }^{7}$ exfoliated deciduous teeth stem cells, ${ }^{8}$ periodontal ligament stem cells, ${ }^{9}$ apical papilla stem cells,${ }^{10}$ dental follicle stem cells, ${ }^{11}$ and gingiva stem cells. ${ }^{12,13}$ Human periodontal ligament stem cells (hPDLSCs) displayed substantial proliferation capacity ex vivo ${ }^{14,15}$ and mesengenic differentiation capacity. ${ }^{16}$

Various bacteria and their pathogen associated molecules including lipopolysaccharides (LPS) activate a cascade of chronic inflammatory events and modulate the host tissue response. Many types of gram-negative anaerobic bacteria are involved in periodontitis. Porphyromonas gingivalis is the major periodontitis pathogen, that triggers initiation and progression of periodontal diseases. ${ }^{17,18}$ In addition to bacteria, genetics and environmental factors also play a crucial role in the etiology of periodontitis via regulating epigenetic modifications. ${ }^{19}$ Bacteria and their products can produce alterations in DNA methylation, which modifies the regulation of inflammatory genes followed by disease progression..$^{20-22}$ DNA methylation and histone acetylation are the major epigenetic modifications induced by diseases and environmental factors. $^{23,24}$ DNA (cytosine-5) methyltransferase 1 (DNMT1) and histone deacetylases (HDACs) are the key controllers, which regulate DNA methylation and histone acetylation, respectively. ${ }^{25}$
Correspondence: Oriana Trubiani, Department of Medical, Oral and Biotechnological Sciences, University "G. d'Annunzio" ChietiPescara, Via dei Vestini 31, 66100 Chieti, Italy.

Fax: +39.0871.3554033

E-mail: trubiani@unich.it

Key words: Periodontitis; inflammation; human periodontal mesenchymal stem cells; epigenetic regulation; DNMT1; p300; Alzheimer's disease.

Acknowledgments: this work has been supported with OT ex $60 \%$ funds, University "G. d'Annunzio".

Contributions: FD, SRT, IM, experiments performing; MDO, samples collection; FD, SRT, MDO, data analysis, manuscript revision; EM, OT manuscript drafting; PB, EM, OT, study design. All authors have edited, read and approved the final manuscript.

Conflict of interest: the authors declare no conflict of interest.

Received for publication: 9 June 2017. Accepted for publication: 2 August 2017.

This work is licensed under a Creative Commons Attribution-NonCommercial 4.0 International License (CC BY-NC 4.0).

(C) Copyright F. Diomede et al., 2017

Licensee PAGEPress, Italy

European Journal of Histochemistry 2017; 61:2826 doi:10.4081/ejh.2017.2826

In periodontal disease condition, histone acetylation promotes the transcription of inflammatory genes such as p300/CBP histone acetyltransferase, NF-kB and other proinflammatory cytokines. ${ }^{26}$ However, the impact of histone modifications during the progression of periodontitis remains unclear. NF-kB signaling pathway could be involved in sustained histone modifications which further augments the disease progression. ${ }^{27}$ Nuclear transcription factor NF-kB has a key role to activate innate immunity which causes osteoclast differentiation and to induce bone resorption. ${ }^{28}$ DNA methylation is regulated by two different types of DNA methyltransferases (DNMTs): de novo methyltransferases (DNMT3a and DNMT3b), which are active during early development ${ }^{29}$ and maintenance methyltransferase (DNMT1), which regulates methylated and unmethylated $\mathrm{CpG}$ sites in the cells. ${ }^{30-32}$

In the present study, we have investigated the epigenetic modifications elicited by Porphyromonas gingivalis LPS (LPS-G) using hPDLSCs as a model system to study novel 
biomarkers linked to this oral inflammatory disease. To this end, we have examined the expression of DNMT1, p300 and NF-kB followed by LPS-G treatment in hPDLSCs.

\section{Materials and Methods}

\section{Ethic statement}

The present study was approved by the Medical Ethics Committee at the Medical School, "G. d'Annunzio" University, Chieti, Italy (n. 266/17.04.14). All healthy volunteers enrolled in this study have signed the informative consent form. The Department of Medical, Oral and Biotechnological Sciences and the Laboratory of Stem Cells and Regenerative Medicine are certified according to the quality standard ISO 9001:2008 (certificate n. $32031 / 15 / \mathrm{S}$ ).

\section{Cell culture}

Periodontal ligament biopsies were collected from premolar teeth of healthy volunteers. All patients provided written informed consent to participate in the study. Before the biopsy collection, each patient was pre-treated for one week with professional dental hygiene and chlorhexidine. Explants were obtained from alveolar crest and horizontal fibers of the periodontal ligament by scraping the roots using a Gracey's curette. ${ }^{33}$ Periodontal tissue fragments were cut, washed with PBS (Lonza, Basel, Switzerland) and placed in a TheraPEAK ${ }^{\text {TMMSCGM-CD }}{ }^{\text {TM }}$ Bullet Kit serum free, chemically defined (MSCGMCD) medium (Lonza) at $37^{\circ} \mathrm{C}$ for the growth of human MSCs. Cells spontaneously migrated from the explants after reaching about $80 \%$ of confluence were trypsinized (LiStar Fish, Milan, Italy), and subsequently subcultured until passage 2 (P2). Cells utilized for the experimental assays were at $\mathrm{P} 2$.

\section{LPS-G treatment}

hPDLSCs were divided in two groups: group 1, untreated control (hPDLSCs); and group 2 , cells treated with $5 \mu \mathrm{g} / \mathrm{mL}$ LPS-G (InvivoGen, San Diego, CA, USA) (hPDLSCs/LPS-G) for $24 \mathrm{~h}$.

\section{Morphological evaluation}

After $24 \mathrm{~h}$, hPDLSCs and hPDLSCs treated with LPS-G were fixed with $2.5 \%$ glutaraldehyde in $0.1 \mathrm{M}$ cacodylate buffer $\mathrm{pH} 7.4$ for $2 \mathrm{~h}$, stained with toluidine blue solution and observed by inverted optical microscope Leica DMIL (Leica Microsystems, Milan, Italy).

\section{MTT assay}

Cell viability was evaluated by 3-(4,5dimethyl-2-thiazolyl)-2,5-diphenyl-2-Htetrazolium bromide (MTT) test. $1.5 \times 10^{4}$ cells of each group were plated in 96-well plates and were incubated with $200 \mu \mathrm{lcul}-$ ture medium. After incubation, $20 \mu \mathrm{L}$ MTT solution was added to each well and incubated for $3 \mathrm{~h} .{ }^{34}$ The absorbance was measured on an automated microplate reader (Sinergy HT, Biotek Instruments, Bad Friedrichshall, Germany) at $570 \mathrm{~nm}$.

\section{Immunofluorescence analysis}

hPDLSCs and hPDLSCs/LPS-G were processed as previously reported by Trubiani et al. ${ }^{35}$ The following primary monoclonal antibodies were used: antihuman NF-kB (1:250, rabbit) (OriGene Technologies, Inc., Rockville, MD, USA), anti-DNMT1 (1:250, rabbit) (OriGene), anti-p300 (1:250, rabbit) (OriGene). Then, cells were incubated for $1 \mathrm{~h}$ at $37^{\circ} \mathrm{C}$ with Alexa Fluor 568 red fluorescence conjugated goat anti-rabbit secondary antibodies (1:200) (Molecular Probes). Subsequently, cells were incubated with Alexa Fluor 488 phalloidin green fluorescence conjugate (1:200, Molecular Probes) to mark cytoskeleton actin. Cell nuclei were stained with TOPRO (1:200, Molecular Probes) for $1 \mathrm{~h}$ at $37^{\circ} \mathrm{C}$. Glass coverslips were placed upside down on glass slides and mounted with Prolong antifade (Molecular Probes). ${ }^{36}$ Samples were observed with Zeiss LSM510META confocal system (Zeiss, Jena, Germany) connected to an inverted Zeiss Axiovert 200 microscope equipped with a Plan Neofluar oil-immersion objective $(63 \mathrm{x})$. Images were collected using an argon laser beam with excitation lines at $488 \mathrm{~nm}$ and a helium-neon source at 543 and $665 \mathrm{~nm}$.

\section{Nuclei isolation}

Treated and untreated hPDLSCs $\left(5 \times 10^{6}\right)$ were washed with PBS and resuspended in $500 \mu \mathrm{L}$ of a hypotonic/detergent buffer [10 mM Tris-Cl pH 7.8, 0.125\% Nonidet P-40, $1 \mathrm{mM} \beta$-mercaptoethanol, 1 $\mathrm{mM}$ phenylmethylsulfonyl fluoride, 10 $\mu \mathrm{L} / \mathrm{mL}$ cocktail inhibitor (Protease Inhibitor Cocktail, Sigma-Aldrich, St. Louis, MO, USA), $\quad 0.1 \mu \mathrm{g} / \mathrm{mL}$ soybean trypsin inhibitor]. Cells were sheared by 5 passages through a 22-gauge syringe needle. ${ }^{37}$ Nuclei were recovered by low speed centrifugation $(800 \times \mathrm{g}$ for $10 \mathrm{~min})$ and washed two times in $10 \mathrm{mM}$ Tris- $\mathrm{Cl} \mathrm{pH} \mathrm{7.4,} 2 \mathrm{mM} \mathrm{MgCl} 2$ plus protease inhibitors as mentioned above. The pellet, representing the intact nuclei fraction, was used for biochemical experiments.

\section{Western blot analysis}

Thirty micrograms of proteins from hPDLSCs and hPDLSCs/LPS-G and from extracted nuclei were separated on SDSPAGE and subsequently transferred to nitrocellulose sheets using a semidry blotting apparatus. Sheets were saturated for $120 \mathrm{~min}$ at room temperature in blocking buffer (1xTBS, 5\% milk, 0.1\% Tween-20), then incubated overnight at $4^{\circ} \mathrm{C}$ in blocking buffer containing primary antibodies to NFkB (1:2000, rabbit) (OriGene), DNMT1 (1:1000, rabbit) (OriGene), p300 (1:750, rabbit) (OriGene) and $\beta$-actin (1:750, mouse) (Santa Cruz Biotechnology, Santa Cruz, CA, USA). After four washes in TBS containing $0.1 \%$ Tween-20, samples were incubated for $60 \mathrm{~min}$ at room temperature with peroxidase-conjugated secondary antibody diluted $1: 1000$ in $1 x$ TBS with $2.5 \%$ dry milk and $0.1 \%$ Tween-20. ${ }^{38}$ Bands were visualized by ECL method and quantified by Alliance 2.7 (UVItec Ltd., Cambridge, UK).

\section{Next generation sequencing (NGS)}

Total RNA was isolated using a Reliaprep RNA Cell Miniprep System (Promega, USA). TruSeq RNA Access library kit (Illumina, Inc., San Diego, CA, USA) was applied to make RNA sequencing libraries. Fifty ng of total RNA was fragmented at $94^{\circ} \mathrm{C}$ for $8 \mathrm{~min}$ on a thermal cycler. First strand cDNA synthesis was produced with random hexameres and SuperScript II Reverse Transcriptase (Invitrogen) using the following temperature profile: $25^{\circ} \mathrm{C}$ for $10 \mathrm{~min}, 42^{\circ} \mathrm{C}$ for 15 $\min$ and $70^{\circ} \mathrm{C}$ for $15 \mathrm{~min}$. Then, RNA templates were removed and double stranded cDNA was produced using dUTP. Subsequently, the 3 'ends of the cDNA were adenylated and ligated with indexing adaptor. The following PCR temperature profile was used for the selective enrichment of adaptor-ligated DNA fragments: $98^{\circ} \mathrm{C}$ for $10 \mathrm{~s}, 60^{\circ} \mathrm{C}$ for $30 \mathrm{~s}$ and $72^{\circ} \mathrm{C}$ for $30 \mathrm{~s}(15$ cycles). Then, the libraries were validated and were hybridized with the selective probes to capture exome. Probes with enriched libraries were captured using streptavidin coated magnetic beads, eluted from the beads, cleaned up with AMPure XP beads and processed for the second PCR amplification using following temperature profile: $98^{\circ} \mathrm{C}$ for $10 \mathrm{~s}, 60^{\circ} \mathrm{C}$ for $30 \mathrm{~s}$ and $72^{\circ} \mathrm{C}$ for $30 \mathrm{~s}$ (10 cycles). Later, the libraries were quantified with KAPA Library Quantification Kit-Illumina/ABI Prism ${ }^{\circledR} \quad$ (Kapa Biosystems, Inc., Wilmington, MA, USA) and validated using Agilent High Sensitivity DNA Kit on a Bioanalyzer. DNA fragments were meas- 
ured in the range of 200-650 bp. Peak was observed around $250 \mathrm{bp}$. After normalization to $12 \mathrm{pM}$, DNA libraries were processed for cluster and single read sequencing (150 cycles) using MiSeq tool (Illumina, Inc.). The libraries were loaded on a MiSeq Flow Cell v3 to make clustering and were sequenced. Later, the reads were grouped with specific indexes using demultiplexing software tool CASAVA (ver. 1.8.2, Illumina, Inc.) and were mapped against "Homo sapiens UCSC hg19" reference sequences using RNA-Seq Alignment ver. 1.0.0. TopHat 2 (Bowtie 1) was applied for the Read mapping. Fragments per kilobase of gene model per million mapped reads (FPKM) values were calculated as described by Rajan et al. ${ }^{12}$

\section{Statistical analysis}

All experiments were performed in triplicate. The data are presented as means \pm standard error and were analyzed using SIGMASTAT (SPSS Inc., Chicago, Ill., USA). Oneway repeated-measurement analysis of variance (ANOVA), followed by the post-hoc Holm-Sidak test (when appropriate) and the Student's paired $t$-test were used for statistical analysis. A P-value less than 0.05 was considered statistically significant. In NGS experiment, the statistical analysis on the read counts to measure the proportion of differentially expressed genes between two samples was performed by The Cufflinks Assembly \& DE package ver. 2.0.0. False discovery rate (FDR; Q) value less than 0.05 was considered statistically significant.

\section{Results}

\section{Morphological and viability evaluation}

First, we have examined the morphological changes and viability in hPDLSCs treated with LPS-G. hPDLSCs (Figure 1A) and LPS-G treated hPDLSCs (Figure 1B) showed a fibroblastic-like shape. However, LPS-G treated cells showed long cytoplasmic processes and a low cell density when compared to the control cells. The proliferation rate of hPDLSCs was assessed by means of MTT assay. Obtained data showed a significant exponential cell growth during all endpoint in hPDLSCs, while LPS-G induces a significant reduction in cell viability $(\mathrm{P}<0.01)$ (Figure 1C).

\section{LPS-G induces epigenetic modula- tions in hPDLSCs}

Then, we have investigated the modulations in epigenetic regulation caused by
LPS-G treatment. Expression and subcellular localization of NF-kB, DNMT1 and p300 were observed by immunofluorescence with confocal microscopy. Increased nuclear translocation of NF-kB was observed in LPS-G-treated hPDLSCs (Figure 2 A2), while its predominant cytoplasmic expression of NF-kB was noticed in control untreated cells (Figure 2 A1). Nuclear histone acetyl transferase p 300 was enhanced in hPDLSCs treated with LPS-G (Figure 2 B2), while basal expression was observed in untreated cells (Figure 2 B1). On the contrary, DNMT1 expression was significantly suppressed in LPS-G- treated cells (Figure $2 \mathrm{C} 2$ ), when compared to untreated cells (Figure $2 \mathrm{C} 1$ ). These results were further confirmed with western blot analysis. Total expression of NF-kB was unaltered (Figure 3A), while nuclear expression of NF-kB was markedly increased in LPS-G-treated hPDLSCs, but not in the untreated cells (Figure 3B). Similarly, p300 level was increased in the cells treated with LPS-G (Figure 3D). As expected, DNMT1 was markedly reduced followed by LPS-G treatment (Figure 3C). Densitonetric analysis of specific band provided a protein quantification (Figure $3 \mathrm{~F}$ ).
These results revealed that LPS-G significantly suppressed DNMT1, while upregulated the expression of p300 and NF-kB, which may trigger the transcription of inflammatory genes in hPDLSCs. NGS data supported these results. DNMT1 was downregulated and p300 was upregulated in LPS-G-treated hPDLSCs (Figure 4) $(\mathrm{Q}<0.05)$. Histone deacetylase HDAC1 was reduced while HDAC2 was increased after LPS-G treatment (Figure 4) $(\mathrm{Q}<0.05)$. Moreover, proinflammatory cytokine transcripts, tumor necrosis factor alpha induced protein 1 (TNFAIP1) and interleukin 6 signal transducer (IL6ST) were significantly enhanced in LPS-G-treated hPDLSCs (Figure 3) $(\mathrm{Q}<0.05)$.

\section{LPS-G modulates genes associated with AD in hPDLSCs}

Considering the connection between periodontitis and $\mathrm{AD}$, we have examined the expression of genes linked with $\mathrm{AD}$ in LPS-G treated hPDLSCs. We have noticed that AD-linked genes such as amyloid beta precursor protein (APP), amyloid beta precursor protein binding protein 2 (APPBP2), interferon gamma receptor 1 (IFNGR1), matrix metallopeptidase 1 (MMP1), MMP2
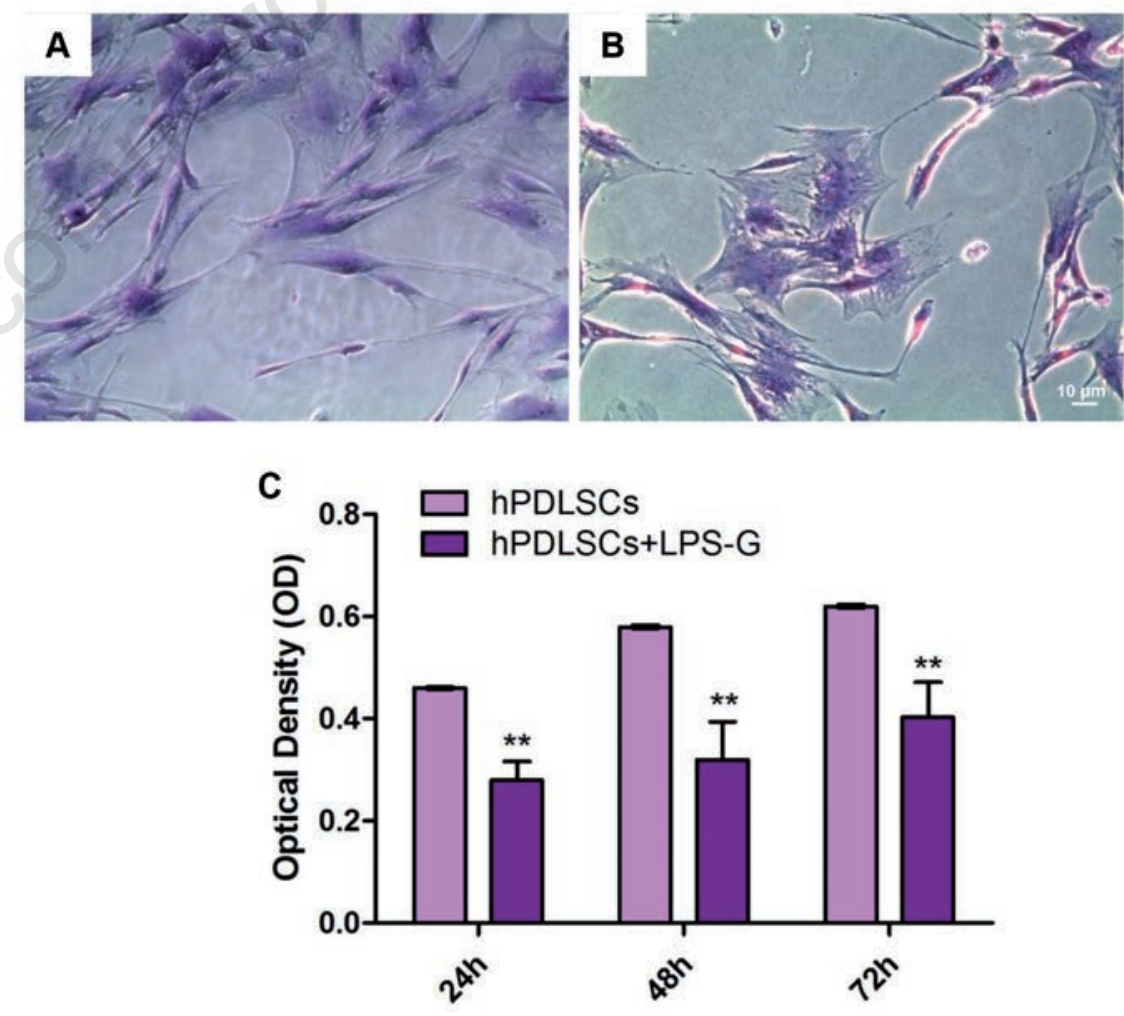

Figure 1. Morphological evaluation. A) Isolation and culture of hPDLSCs from periodontal ligament tissues; cells showed a fibroblastic morphology. B) After 24 h of LPS-G treatment, cells showed a low density and morphological changes, such as long and numerous cytoplasmic processes. C) MTT showed a reduction in cell proliferation rate in all examined endpoint in LPS-G treated cells. Magnification: $10 x ; * P<0.01$. 
and MMP16 were markedly increased in LPS-G-treated hPDLSCs than in control untreated hPDLSCs (Table 1; $\mathrm{Q}<0.0001$ ). Relative $\log 2$ fold change expression of these genes in hPDLSCs and LPS-G-treated hPDLSCs are shown in Table 1. These results suggest that LPS-G-induced inflammation in hPDLSCs may activate the genes associated with $\mathrm{AD}$.

\section{Discussion}

Periodontitis is a prevalent inflammatory disease arising from the gum plaques produced by oral pathogens including Porphyromonas gingivalis. Advanced form of periodontitis results in the retraction of gingiva, loss of periodontal ligament, and degeneration of surrounding alveolar bone. Chronic periodontitis may increase the risk of neuroinflammatory diseases such as AD. ${ }^{39,40}$ Complex molecular mechanisms are involved in the stimulation and maintenance of inflammation, among which epigenetic pathways received special attention because of their upstream regulations. Epigenetic modifications include chemical alterations of DNA and associated proteins, leading to remodeling of the chromatin and activation or inactivation of gene transcription. These changes can contribute to the development and maintenance of cancer, autoimmune and inflammatory diseases, including periodontitis. Interestingly, some epigenetic modifications are reversible and can be stimulated by environmental factors. ${ }^{41,42}$ Knowledge on the modification of epigenetic mechanisms may provide substantial inputs regarding the mechanism of the key regulatory pathways of the genes involved in the maintenance of chronic inflammation. Indeed, the crucial role of DNA and histone modifications, the two major epigenetic regulations, have been described in periodontitis. ${ }^{26}$ Several studies have demonstrated that in periodontal disease, gene expression can be influenced by DNA methylation; ${ }^{26}$ however, only few studies have reported the histone modifica- tions induced by LPS-G stimulus. ${ }^{43}$ Earlier studies have demonstrated that in B cells, LPS-G induced methylation and acetylation in $\mathrm{H} 3$ and $\mathrm{H} 4$ histone proteins. ${ }^{26}$ Recently, it has been demonstrated that periodontitis is
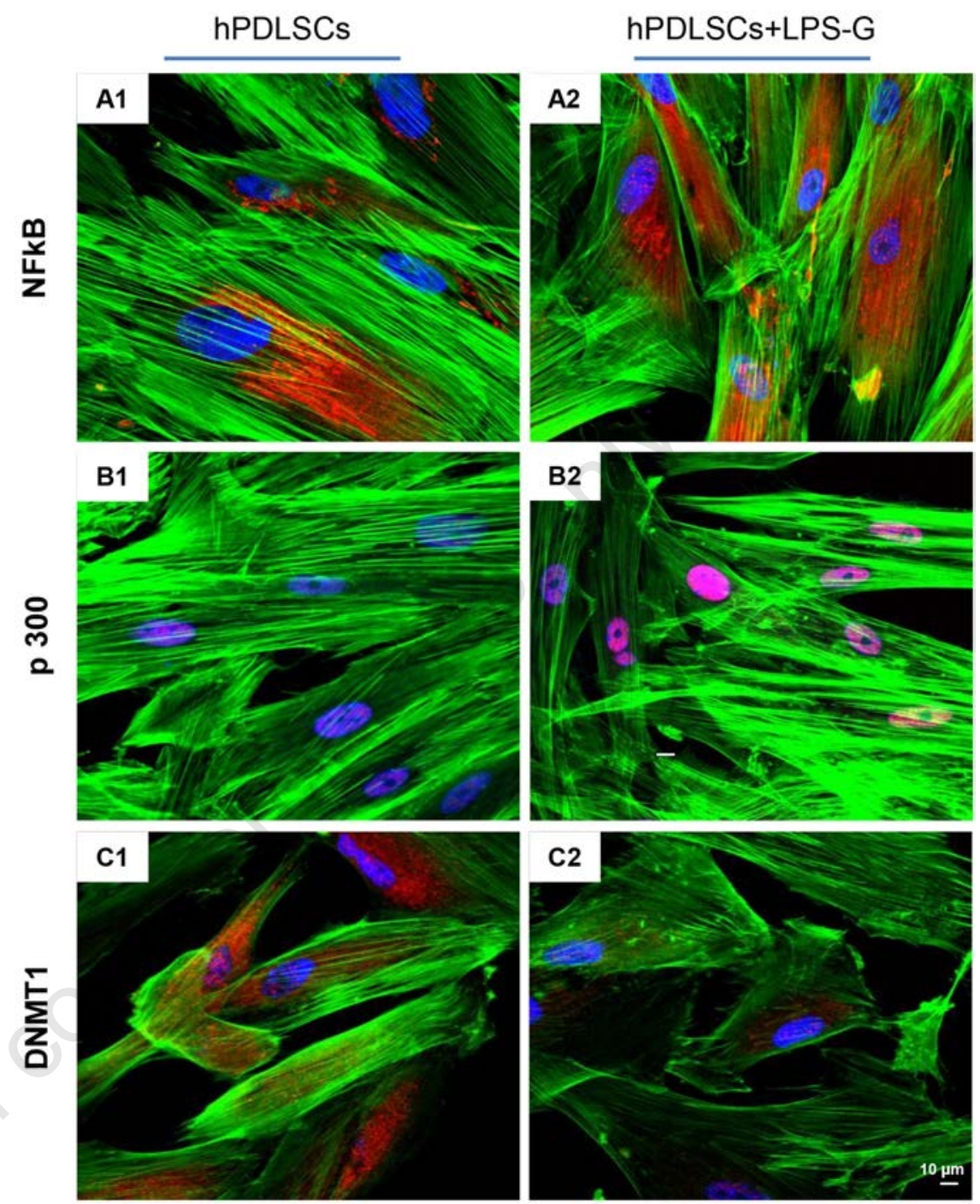

Figure 2. Immunofluorescence. Cells grown on coverslips were fixed and stained with the indicated primary and secondary antibodies and DAPI, and subjected to confocal microscopy. NF-kB expression in hPDLSCs (A1) and in hPDLSCs treated with LPS-G (A2). p300 expression in hPDLSCs (B1) and in hPDLSCs treated with LPS-G (B2). DNMT1 expression in hPDLSCs (C1) and in hPDLSCs treated with LPS-G (C2). Data are representative of three independent experiments. Magnification: 63x.

Table 1. LPS-G modulates genes associated with Alzheimer's disease. NGS data revealed that LPS-G treatment significantly increased the expression of AD-linked genes such as APP, APPBP2, IFNGR1, MMP1, MMP2 and MMP16 in hPDLSCs (Q<0.0001).

\begin{tabular}{lcccc} 
Gene & hPDLSCs & hPDLSCs+LPS-G & log2 fold change & Q-value \\
APP & 405.63 & 1057.55 & 1.38 & 0.0001 \\
APPBP2 & 4.48 & 6.94 & 0.63 & 0.0001 \\
\hline IFNGR1 & 14.82 & 34.78 & 1.23 & 0.0001 \\
MMP1 & 13.84 & 161.31 & 3.54 & 0.0001 \\
\hline MMP2 & 975.87 & 1359.89 & 0.48 & 0.0001 \\
MMP16 & 2.30 & 8.99 & 1.97 & 0.0001 \\
\hline
\end{tabular}




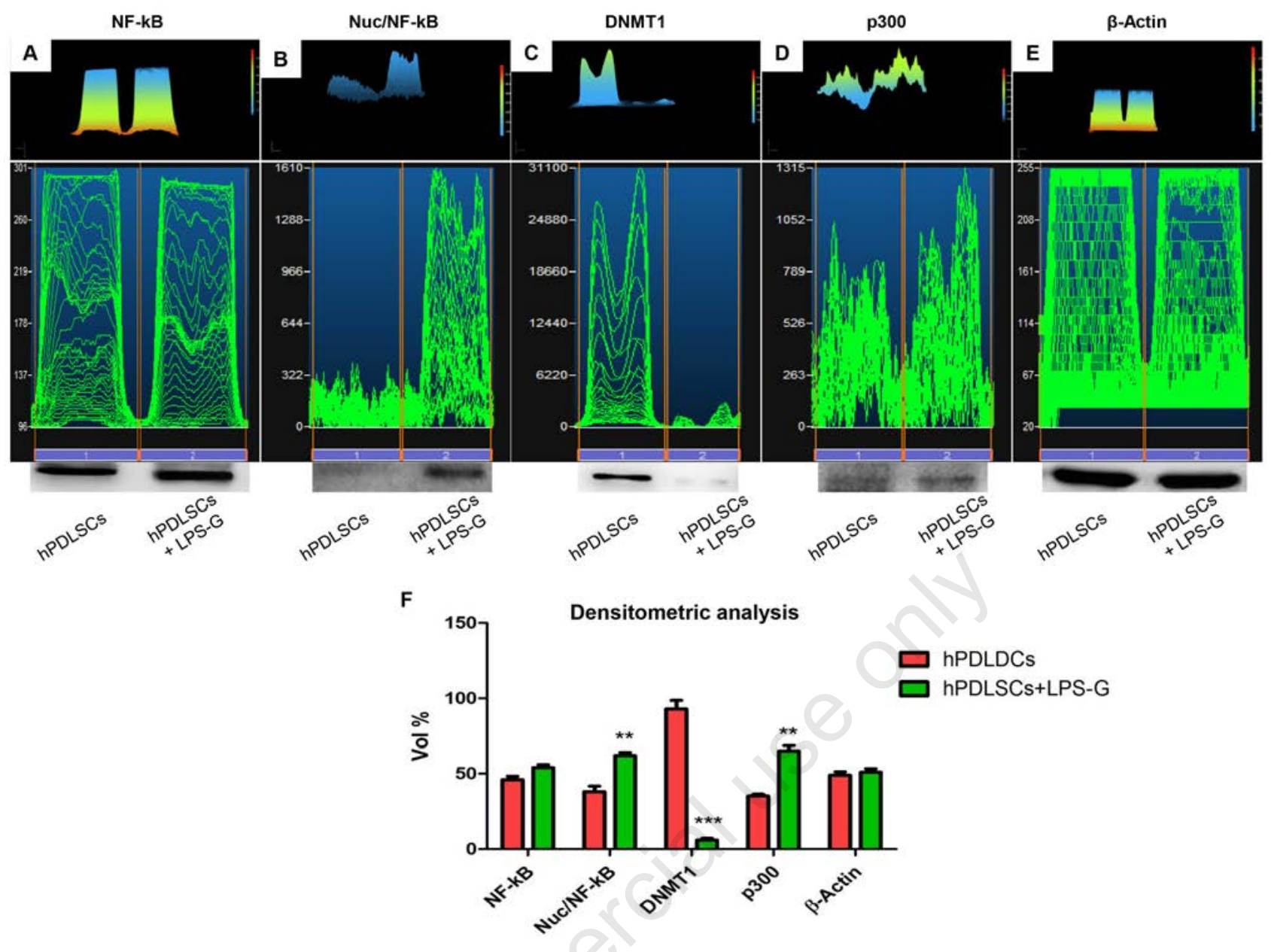

Figure 3. Western blot analysis of NF-KB (A), NF- $\mathrm{KB}$ in nuclear extract (B), DNMT1 (C) and p300 (D) expression in hPDLSCs and in hPDLSCs treated with LPS-G for $24 \mathrm{~h}$. $\beta$-actin was used as a housekeeping protein (E). Densitometric analysis of protein specific bands (F). ${ }^{* *} \mathbf{P}<0.01 ;{ }^{* * *} \mathrm{P}<0.001$.

expression, while hypomethylation pattern is closely associated with transcriptional activation. ${ }^{46}$ Moreover, it has been demonstrated that LPS-G downregulated the expression of DNA methylases DNMT3a and DNMT1 in keratinocytes. ${ }^{44}$

In our recent study we reported that in hPDLSCs, LPS-G treatment activates the TLR4/MyD88 complex, induces NF-kB nuclear translocation and triggers the secretion of proinflammatory cytokines including TNF- $\alpha .{ }^{47}$ Based on these findings, we assume that hPDLSCs may represent an appropriate stem cell modelling through which we may understand the biological machinery of epigenetic system and inflammation in response to LPS-G in periodontal tissues. To this end, we have investigated the expression of DNMT1, p300 and NF-kB in hPDLSCs administered with LPS-G. Immunofluorescence and Western blot results demonstrated that LPS-G treatment significantly suppressed the expression of DNMT1 in hPDLSCs. This result is corrob-

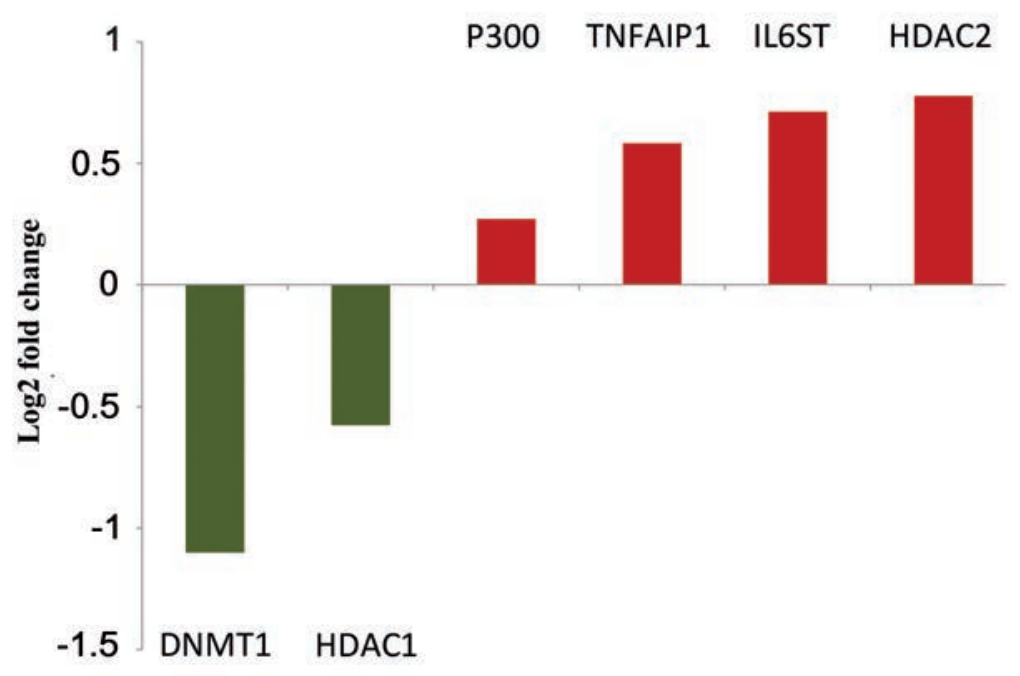

Figure 4. Gene expression. Next generation sequencing demonstrated the modulation of genes expressed in untreated and LPS-G treated hPDLSC. P300, TNFAIP1, IL6ST and HDAC2 2 were expressed greater than 2 -fold $(\log 2$ fold change; $Q<0.05)$. DNMT1 and HDAC1 genes were significantly downregulated in LPS-G stimulated hPDLSCs when compared with untreated cells $(Q<0.05)$. Up-regulated transcripts are highlighted in red color; downregulated transcripts are highlighted in green color. 
orated with the findings from Yin and Chung, ${ }^{48}$ which showed that $\mathrm{P}$. gingivalis (whole bacteria) could cause a decrease in DNMT1 gene expression in oral epithelial cell and that $P$. gingivalis may modulate DNA methylation status of the genes involved in the pathogenesis of periodontitis. Moreover, LPS stimulation down regulated DNMT1 expression in human $\mathrm{HaCaT}$ keratinocytes and in oral ephitelial cells. ${ }^{44}$

LPS triggers many intracellular signaling cascades among which NF-kB pathway is a crucial one. ${ }^{49,50} \mathrm{NF}-\mathrm{kB}$ represents a family of transcription factors essential for the induction of the most important classes of inflammatory genes, including genes involved in periodontitis progression. Both recruitment of NF-kB to target promoters and NF-kB-induced transcriptional genes could be modulated through chromatin modification. ${ }^{50} \mathrm{CBP} / \mathrm{p} 300$ are general transcriptional co-activators that help NF-kB to bridge with the basal transcription machinery. ${ }^{51} \mathrm{CBP} / \mathrm{p} 300$ interact with a large array of transcription factors to integrate multiple cellular signaling pathways. ${ }^{52,53}$ In addition, $\mathrm{CBP} / \mathrm{p} 300$ possess chromatin-remodeling capabilities owing to their histone- and transcription factor-acetylating properties. p300 is not only a transcriptional adaptor but also a histone acetyltransferase. ${ }^{54}$ In our hPDLSCS-based stem cell model, we found that LPS-G increased the expression of NF$\mathrm{kB}$ and its coactivator $\mathrm{p} 300$, indicating the critical regulatory effect of these molecules during the inflammation process triggered by LPS-G. Moreover, we noticed that histone deacetylase HDAC1 was decreased while HDAC2 was increased followed by LPS-G treatment. These results suggested the differential regulatory effect of these deacetylases over epigenetics regulation induced by LPS-G. In addition, NGS data revealed the upregulation of proinflammatory cytokine transcripts TNFAIP1 and IL6ST in LPS-G-treated hPDLSCs, which suggested the activation of inflammatory cytokines followed by LPS-G.

Emerging evidence suggests that poor oral health influences the initiation and/or progression of diseases such as atherosclerosis (with sequelae including myocardial infarction and stoke), diabetes mellitus and neurodegenerative diseases. ${ }^{55}$ In particular, chronic periodontitis is associated with an increase in cognitive decline in Alzheimer's disease. ${ }^{5}$ Considering the association between periodontitis and $\mathrm{AD}$, in the present study we have examined if LPS-G treatment may modulate the genes linked with $\mathrm{AD}$ in hPDLSCs. NGS data showed that genes involved in $\mathrm{AD}$ pathogenesis such as APP, APPBP2, IFNGR1, MMP1, MMP2 and MMP16 were significantly upregulated in LPS-G-treated hPDLSCs. These data support the notion that periodontitis may increase the risk of $\mathrm{AD}$. We assume that LPS-G-treated hPDLSCs might be a potential in vitro model system to explore the link between periodontitis and AD. The importance of epigenetics in periodontal disease has been studied with growing interest in the last few years due to its strict correlation to the systemic diseases Recent advances in epigenomic approaches allow mapping of the methylation state in the genome, which may help to identify new biomarkers as well as to develop novel therapeutic strategies for preventing periodontal disease.

In conclusion, we demonstrated the activation of epigenetic modifications in LPS-G-treated hPDLSCs. Reduced DNMT1 and increased NF-kB and p300 were noticed in hPDLSCs treated with LPSG. We proposed that hPDLSCs-based stem cell model could be a potential in vitro tool to evaluate epigenetic changes associated with periodontitis.

\section{References}

1. Demmer RT, Behle JH, Wolf DL, Handfield M, Kebschull M, Celenti R, et al. Transcriptomes in healthy and diseased gingival tissues. J Periodontol 2008;79:2112-24.

2. Kornman KS. Mapping the pathogenesis of periodontitis: a new look. J Periodontol 2008;79:1560-8.

3. Sochocka M, Sobczynski M, SenderJaneczek A, Zwolinska K, Blachowicz $\mathrm{O}$, Tomczyk $\mathrm{T}$, et al. Association between periodontal health status and cognitive abilities. The role of cytokine profile and systemic inflammation. Curr Alzheimer Res 2017 doi: 10.2174 /1567205014666170316163340 [Epub ahead of print]

4. Lee YT, Lee HC, Hu CJ, Huang LK, Chao SP, Lin CP, et al. Periodontitis as a Modifiable risk factor for dementia: A nationwide population-based cohort study. J J Am Geriatr Soc 2017;65:301-5.

5. Ide M, Harris M, Stevens A, Sussams R, Hopkins V, Culliford D, et al. Periodontitis and Cognitive Decline in Alzheimer's Disease. PloS One 2016;11:e0151081.

6. Chatzivasileiou K, Kriebel K, Steinhoff G, Kreikemeyer B, Lang H. Do oral bacteria alter the regenerative potential of stem cells? A concise review. J Cell Mol Med 2015;19:2067-74.

7. Diomede F, Caputi S, Merciaro I, Frisone S, D'Arcangelo C, Piattelli A, et al. Pro-inflammatory cytokine release and cell growth inhibition in primary human oral cells after exposure to endodontic sealer. Int Endod J 2014;47: 864-72.

8. Miura M, Gronthos S, Zhao M, Lu B, Fisher LW, Robey PG, et al. SHED: stem cells from human exfoliated deciduous teeth. Proc Natl Acad Sci USA 2003;100:5807-12.

9. Rajan TS, Giacoppo S, Diomede F, Ballerini P, Paolantonio M, Marchisio $\mathrm{M}$, et al. The secretome of periodontal ligament stem cells from MS patients protects against EAE. Sci Rep 2016;6: 38743.

10. Sonoyama W, Liu Y, Fang D, Yamaza T, Seo BM, Zhang C, et al. Mesenchymal stem cell-mediated functional tooth regeneration in swine. PloS One 2006; 1:e79.

11. Morsczeck C, Gotz W, Schierholz J, Zeilhofer F, Kuhn U, Mohl C, et al. Isolation of precursor cells (PCs) from human dental follicle of wisdom teeth. Matrix Biol 2005;24:155-65.

12. Rajan TS, Scionti D, Diomede F, Grassi G, Pollastro F, Piattelli A, et al. Gingival stromal cells as an in vitro model: Cannabidiol modulates genes linked with amyotrophic lateral sclerosis. J Cell Biochem 2017;118:819-28.

13. Trubiani O, Toniato E, Di Iorio D, Diomede F, Merciaro I, C DA, et al. Morphological analysis and interleukin release in human gingival fibroblasts seeded on different denture base acrylic resins. Int J Immunopathol Pharmacol 2012;25:637-43.

14. Rajan TS, Giacoppo S, Trubiani O, Diomede F, Piattelli A, Bramanti P, et al. Conditioned medium of periodontal ligament mesenchymal stem cells exert antiinflammatory effects in lipopolysaccharide-activated mouse motoneurons. Exp Cell Res 2016;349:152-61.

15. Diomede F, Zini N, Gatta V, Fulle S, Merciaro I, D'Aurora M, et al. Human periodontal ligament stem cells cultured onto cortico-cancellous scaffold drive bone regenerative process. Eur Cell Mater 2016;32:181-201.

16. Libro R, Scionti D, Diomede F, Marchisio M, Grassi G, Pollastro F, et al. Cannabidiol modulates the immunophenotype and inhibits the activation of the inflammasome in human gingival mesenchymal stem cells. Front Physiol 2016;7:559.

17. Zhang P, Liu JZ, Xu QA, Harber G, Feng $\mathrm{X}$, Michalek SM, et al. TLR2-dependent modulation of osteoclastogenesis by Porphyromonas gingivalis through differ- 
ential induction of NFATc1 and NF-kappa B. J Biol Chem 2011;286:24159-69.

18. Lamont RJ, Jenkinson HF. Life below the gum line: Pathogenic mechanisms of Porphyromonas gingivalis. Microbiol Mol Biol Rev 1998;62:1244-63

19. Offenbacher S, Barros SP, Beck JD. Rethinking periodontal inflammation. J Periodontol 2008;79(8 Suppl):1577-84.

20. Fraga MF, Ballestar E, Villar-Garea A, Boix-Chornet M, Espada J, Schotta G, et al. Loss of acetylation at Lys16 and trimethylation at Lys20 of histone $\mathrm{H} 4$ is a common hallmark of human cancer. Nat Gene 2005;37:391-400.

21. Hur K, Niwa T, Toyoda T, Tsukamoto T, Tatematsu M, Yang HK, et al. Insufficient role of cell proliferation in aberrant DNA methylation induction and involvement of specific types of inflammation. Carcinogenesis 2011;32:35-41.

22. Bobetsis YA, Barros SP, Lin DM, Weidman JR, Dolinoy DC, Jirtle RL, et al. Bacterial infection promotes DNA hypermethylation. J Dent Res 2007;86: 169-74.

23. Lod S, Johansson T, Abrahamsson KH, Larsson L. The influence of epigenetics in relation to oral health. Int J Dent Hyg 2014;12:48-54.

24. Sadakierska-Chudy A, Kostrzewa RM, Filip M. A comprehensive view of the epigenetic landscape Part I: DNA methylation, passive and active DNA demethylation pathways and histone variants. Neurotox Res 2015;27:84-97.

25. Kim Y, Kim K, Park D, Lee E, Lee H, Lee YS, et al. DNA methyl transferase I acts as a negative regulator of allergic skin inflammation. Mol Immunol 2013;53:1-14.

26. Martins MD, Jiao Y, Larsson L, Almeida LO, Garaicoa-Pazmino C, Le JM, et al. Epigenetic Modifications of Histones in Periodontal Disease. J Dent Res 2016; 95:215-22.

27. Dev A, Iyer S, Razani B, Cheng G. NFkappaB and innate immunity. Curr Top Microbiol Immunol 2011;349:115-43.

28. Sanchez-Pernaute O, Ospelt C, Neidhart M, Gay S. Epigenetic clues to rheumatoid arthritis. J Autoimmun 2008;30:12-20.

29. Reik W, Kelsey G, Walter J. Dissecting de novo methylation. Nat Genet 1999;23:380-2.

30. Colot V, Rossignol JL. Eukaryotic DNA methylation as an evolutionary device. Bioessays 1999;21:402-11.

31. Bird A. DNA methylation patterns and epigenetic memory. Genes Dev 2002; 16:6-21.
32. Vaissiere T, Sawan C, Herceg Z. Epigenetic interplay between histone modifications and DNA methylation in gene silencing. Mutat Res 2008;659:40-8.

33. Diomede F, Rajan TS, Gatta V, D'Aurora M, Merciaro I, Marchisio M, et al. Stemness maintenance properties in human oral stem cells after long-term passage. Stem Cells Int 2017;2017:5651287.

34. Soundara Rajan T, Giacoppo S, Scionti D, Diomede F, Grassi G, Pollastro F, et al. Cannabidiol activates neuronal precursor genes in human gingival mesenchymal stromal cells. J Cell Biochem 2017;118:1531-46.

35. Trubiani O, Guarnieri S, Diomede F, Mariggio MA, Merciaro I, Morabito C, et al. Nuclear translocation of PKCalpha isoenzyme is involved in neurogenic commitment of human neural crestderived periodontal ligament stem cells. Cell Signal 2016;28:1631-41.

36. Carotti S, Perrone G, Amato M, Gentilucci UV, Righi D, Francesconi M, et al. Reelin expression in human liver of patients with chronic hepatitis $\mathrm{C}$ infection. Eur J Histochem 2017;61:2745.

37. Orciani M, Trubiani O, Guarnieri S, Ferrero E, Di Primio R. CD38 is constitutively expressed in the nucleus of human hematopoietic cells. J Cell Biochem 2008;105:905-12.

38. Verrucci M, Pancrazzi A, Aracil M, Martelli F, Guglielmelli P, Zingariello M, et al. CXCR4-independent rescue of the myeloproliferative defect of the Gata1low myelofibrosis mouse model by Aplidin. J Cell Physiol 2010;225:490-9.

39. Gaur S, Agnihotri R. Alzheimer's disease and chronic periodontitis: Is there an association? Geriatr Gerontol Int 2015;15:391-404.

40. Ganesh P, Karthikeyan R, Muthukumaraswamy A, Anand J. A Potential role of periodontal inflammation in Alzheimer's Disease: A review. Oral Hlth Prev Dent 2017;15:7-12.

41. Wilson AG. Epigenetic regulation of gene expression in the inflammatory response and relevance to common diseases. J Periodontol 2008;79(8 Suppl):1514-9.

42. Barros SP, Offenbacher S. Epigenetics: connecting environment and genotype to phenotype and disease. J Dent Res. 2009;88:400-8.

43. Lod S, Johansson T, Abrahamsson KH, Larsson L. The influence of epigenetics in relation to oral health. Int J Dent Hyg 2014;12:48-54.
44. de Camargo Pereira G, Guimaraes GN, Planello AC, Santamaria MP, de Souza AP, Line SR, et al. Porphyromonas gingivalis LPS stimulation downregulates DNMT1, DNMT3a, and JMJD3 gene expression levels in human $\mathrm{HaCaT}$ keratinocytes. Clin Oral Investig 2013;17:1279-85.

45. Gomez RS, Dutra WO, Moreira PR. Epigenetics and periodontal disease: future perspectives. Inflamm Res 2009; 58:625-9.

46. Jones PA, Laird PW. Cancer epigenetics comes of age. Nat Gen 1999;21:163-7.

47. Diomede F, Zingariello M, Cavalcanti M, Merciaro I, Pizzicannella J, De Isla $\mathrm{N}$, et al. MyD88/ERK/NFkB pathways and pro-inflammatory cytokines release in periodontal ligament stem cells stimulated by Porphyromonas gingivalis. Eur J Histochem 2017;61:2791.

48. Yin L, Chung WO. Epigenetic regulation of human beta-defensin 2 and $\mathrm{CC}$ chemokine ligand 20 expression in gingival epithelial cells in response to oral bacteria. Mucosal Immunol 2011;4:409-19.

49. Jain S, Darveau RP. Contribution of Porphyromonas gingivalis lipopolysaccharide to periodontitis. Periodontol 2000 2010;54:53-70.

50. Kraus D, Winter J, Jepsen S, Jager A, Meyer R, Deschner J. Interactions of adiponectin and lipopolysaccharide from Porphyromonas gingivalis on human oral epithelial cells. PloS One 2012;7: e30716.

51. Merika M, Williams AJ, Chen GY, Collins T, Thanos D. Recruitment of $\mathrm{CBP} / \mathrm{p} 300$ by the IFN beta enhanceosome is required for synergistic activation of transcription. Mol Cell 1998; 1:277-87.

52. Blobel GA. CREB-binding protein and p300: molecular integrators of hematopoietic transcription. Blood 2000; 95:745-55.

53. Kamei Y, Xu L, Heinzel T, Torchia J, Kurokawa R, Gloss B, et al. A CBP integrator complex mediates transcriptional activation and AP-1 inhibition by nuclear receptors. Cell 1996;85:403-14.

54. Ogryzko VV, Schiltz RL, Russanova V, Howard BH, Nakatani Y. The transcriptional coactivators p300 and CBP are histone acetyltransferases. Cell 1996;87: 953-9.

55. Scannapieco FA, Cantos A. Oral inflammation and infection, and chronic medical diseases: implications for the elderly. Periodontol 2000 2016;72:153-75. 